\title{
A phase trajectories optimization method for CPM signal based on Pan-function model
}

\author{
Wei Xue ${ }^{1{ }^{*}}$, Wenjing Shang ${ }^{1+}$, Sergey B. Makarov ${ }^{2}$ and Yidong $X u^{1}$
}

\begin{abstract}
The continuous phase modulation (CPM) signal has the characteristics of continuous phase, excellent spectral properties, less out-band radiation, and constant envelope. Thus, CPM technology is widely used in communication systems. The shape of frequency pulse can influence the bandwidth occupancy of CPM, and smoother phase trajectories are obtained by using smoother frequency pulses. In this paper, a mathematical Pan-function model of the optimized frequency pulse is established and solved by introducing Fourier series, which can provide smooth phase trajectories of CPM signal. The simulations of the CPM signal quadrature modulation and coherent demodulation are performed using the MATLAB software. Moreover, the spectral characteristics of the obtained optimized CPM signal were analyzed and compared with the minimum shift keying (MSK) signal and other CPM signals with smooth frequency pulses. The simulation results indicate that the proposed method provides an excellent bandwidth efficiency compared to other existing methods discussed in this paper. The whole system has been successfully downloaded to field programmable gate array (FPGA) devices. The operating results are consistent with expected results, verifying the correctness of this method.
\end{abstract}

Keywords: CPM, Optimization, Fourier series, Pan function, Frequency pulse shaping

\section{Introduction}

The spectral characteristics of the digitally modulated signals are extremely important in the design of modern digital communication system. Continuous phase modulation (CPM) is one of the most important modulation techniques due to its efficient use of bandwidth. It has been widely applied in many communications, where multipath fading and nonlinear distortions make constant signal envelope and efficient bandwidth necessary.

For CPM systems, the transmitted signal can be expressed in the following form [1]

$$
s(t ; \vec{a})=\sqrt{\frac{2 E_{s}}{T}} \cos \left[2 \pi f_{c} t+\varphi(t ; \vec{a})+\varphi_{0}\right], \quad t \geq 0
$$

*Correspondence: xuewei@hrbeu.edu.cn

${ }^{\dagger}$ Equal contributors

${ }^{1}$ College of Information and Communication Engineering, Harbin Engineering University, 150001, Harbin, China

Full list of author information is available at the end of the article where the information carrying phase is

$$
\varphi(t ; \vec{a})=2 \pi h \sum_{i=-\infty}^{n} a_{i} q(t-i T), \quad n T \leq t \leq(n+1) T
$$

and $\left\{a_{i}\right\}$ is the sequence of $M$-ary information symbols selected from the alphabet $\pm 1, \pm 3, \cdots, \pm(M-1), E_{s}$ is the symbol energy, $T$ is the symbol time, $f_{c}$ is the carrier frequency, and $\varphi_{0}$ is the initial phase of the carrier that can be set to zero in the case of coherent transmission without any loss of generality. $h$ is the modulation index, and the amplitude of the pulse $g(t)$ is chosen to give the maximum phase change $a h \pi$ radians over each symbol interval. The phase pulse $q(t)$ can be represented in general as the time integral of pulse $g(t)$, as given in (3)

$$
q(t)=\int_{0}^{t} g(\tau) d \tau
$$

If $g(t)=0$ for $t>T$, the CPM signal is called full response CPM. If $g(t) \neq 0$ for $t>T$, the modulated 
signal is called partial response CPM. An infinite variety of CPM signals can be generated by choosing different pulse shapes $g(t)$ and by varying the modulation index $h$ and alphabet size $M$. The rectangular pulse, raised cosine pulse, and Gaussian minimum-shift keying (GMSK) are commonly used CPM pulse shapes [2-4]. An appropriate pulse $g(t)$ can be selected that can provide a continuous phase trajectory of modulated signal. This study aimed to achieve an optimized frequency pulse based on Pan-function model, for $h=1 / 2$ binary full response CPM, with characteristics of a higher spectral roll-off rate, less out-of-band radiation, and a constant envelope.

\section{Foundation of the optimized mathematical model}

2.1 Minimum energy radiation criterion outside the frequency bands

The optimized mathematical model is established by introducing the minimum out-of-band radiation criterion [5] that can be expressed as follows:

$$
J=\frac{1}{2 \pi} \int_{-\infty}^{+\infty} g(\omega)|S(\omega)|^{2} d \omega
$$

where the Fourier transform of $a(t)$ is

$$
S(\omega)=\int_{-T / 2}^{T / 2} a(t) e^{-j \omega t} d t
$$

$a(t)$ is the symbol signal waveform, which is an even function assumed in interval $[-T / 2, T / 2], T$ is the symbol period, $|S(\omega)|^{2}$ is the power spectrum of $a(t)$, and $g(\omega)$ is an increasing function that determines the roll-off rate of the spectral density function $S(\omega)$. The increasing function $g(\omega)=\omega^{2 n}(\omega>0)$ with different $n$ values is shown in Fig. 1. The value of $n$ depends on the degree of suppressed out-band radiation. In order to achieve a convergent and integrable $J$, the roll-off rate of $|S(\omega)|^{2}$ should be greater than the increase rate of $g(\omega)$ to limit the roll-off rate of the power spectrum $|S(\omega)|^{2}$ of the signal $a(t)$.

The power spectral density of $a(t)$ can be written as follows:

$$
|S(\omega)|^{2}=\int_{-T / 2}^{T / 2} \int_{-T / 2}^{T / 2} a(t) a(s) e^{-j \omega(t-s)} d t d s
$$

Replacing (6) into (4), and after some manipulations, the following is obtained:

$$
J=\frac{1}{2 \pi} \int_{-\infty}^{+\infty} \int_{-T / 2}^{T / 2} \int_{-T / 2}^{T / 2} g(\omega) a(t) a(s) e^{-j \omega(t-s)} d t d s d \omega
$$

In order to facilitate the solution, the function $g(\omega)$ can be expressed as follows:

$$
g(\omega)=\lim _{r \rightarrow 0} g(\omega) e^{-r \omega}=\lim _{r \rightarrow 0} \omega^{2 n} e^{-r \omega} \quad(r \geq 0)
$$

When $r$ lies on interval $[0,+\infty]$, the integral

$$
\int_{-\infty}^{+\infty} \omega^{2 n} e^{-r \omega} e^{-j \omega(t-s)} d \omega
$$

is convergent; therefore, the order of the integral can be exchanged as follows:

$$
J=\frac{1}{2 \pi} \lim _{r \rightarrow 0} \int_{-T / 2}^{T / 2} \int_{T / 2}^{T / 2} a(t) a(s)\left[\int_{-\infty}^{+\infty} \omega^{2 n} e^{-r \omega} e^{-j \omega(t-s)} d \omega\right] d t d s
$$

Based on the Taylor series expansion,

$$
e^{-r \omega}=1-r \omega+\left(\frac{r \omega}{2 !}\right)^{2}+\ldots \ldots+\frac{(-r \omega) !}{n !}+\ldots
$$

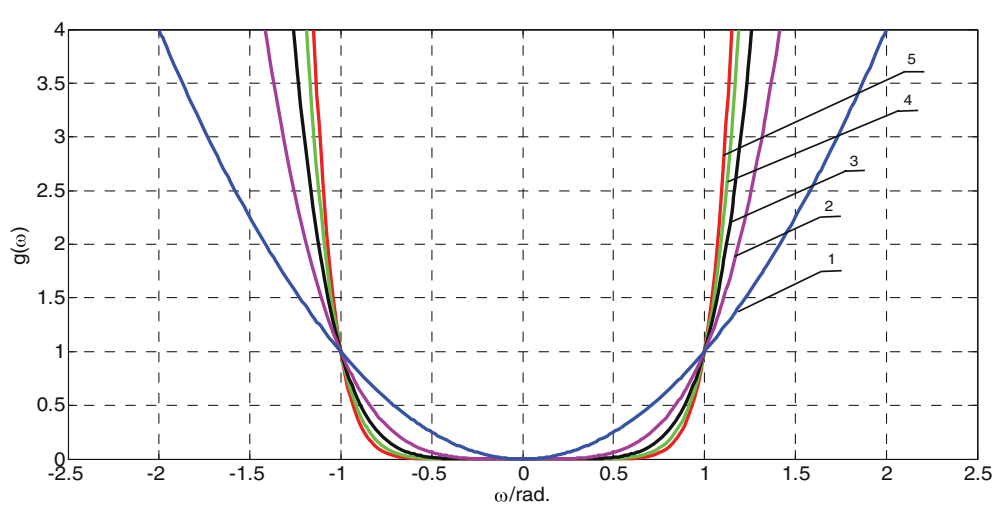

Fig. 1 Waveforms of $g(\omega)$ under different values of $n$ 
According to inverse Fourier transform [6], $\delta(t)$ can be written as follows:

$$
\delta(t)=\frac{1}{2 \pi} \int_{-\infty}^{+\infty} e^{j \omega t} d \omega
$$

As $\delta(t)$ is an even function

$$
\begin{gathered}
\delta(t)=\frac{1}{2 \pi} \int_{-\infty}^{+\infty} e^{-j \omega t} d \omega \\
\delta(t-s)=\frac{1}{2 \pi} \int_{-\infty}^{+\infty} e^{-j \omega(t-s)} d \omega
\end{gathered}
$$

Hence, the following equation can be obtained:

$$
\frac{\partial^{2 n}(\delta(t-s))}{\partial t^{2 n}}=\delta^{(2 n)}(t-s)=\frac{(-j)^{2 n}}{2 \pi} \int_{-\infty}^{+\infty} \omega^{2 n} e^{-j \omega(t-s)} d \omega
$$

when $r \rightarrow 0^{+}$, substituting (12) and (13) into (9), the following expression can be obtained:

$$
\begin{aligned}
J & =\int_{-T / 2}^{T / 2} \int_{-T / 2}^{T / 2} a(t) a(s)(-1)^{n} \delta^{(2 n)}(t-s) d t d s \\
& =(-1)^{n} \int_{-T / 2}^{T / 2} a(t)\left[\int_{-T / 2}^{T / 2} \delta^{(2 n)}(t-s) a(s) d s\right] d t
\end{aligned}
$$

Using the characteristics of $\delta(t)$ function, (14) can be written as follows:

$$
J=(-1)^{n} \int_{-T / 2}^{T / 2} a(t) a^{(2 n)}(t) d t
$$

where $a^{(2 n)}(t)$ is the 2 n-order derivative of the symbol signal.

According to (15), the criterion for minimum out-band radiation can be transformed into the problem of unraveling the symbol function $a(t)$ when minimizing the Pan-functional $J$ [7].

\subsection{Additional constraints of Pan-function}

The mathematical Pan-function is added to the constraints of energy of a single-symbol signal, the boundary conditions, and peak-to-average power ratio (PAPR) of signal.

\subsubsection{Restriction on the symbol boundary condition}

According to [8], assuming that function $a(t)$ is in the finite interval $[-T / 2, T / 2]$, if $a(t), a^{\prime}(t)$, $a^{\prime \prime}(t), \ldots, a^{(n-1)}(t)$ are continuous in interval $[-T / 2, T / 2]$ without skipping, and $a^{(n)}(t)$ is finite, then there is no skipping in the end point of the interval. The Fourier transform of $a(t)$ decays not less than $C / \omega^{n+1}$ in the finite interval $[-T / 2, T / 2]$, where $C$ is a constant. Herein, $a(t)$ should satisfy the following boundary condition:

$a( \pm T / 2)=a( \pm T / 2)^{\prime}=\ldots=a^{(\mathrm{n}-1)}( \pm T / 2)=0$

\subsubsection{Restriction on the single-symbol signal energy condition}

The energy per transmission for the signal $a(t)$ in the finite interval $[-T / 2, T / 2]$ with a length of $T$, can be expressed as follows:

$$
E=\int_{-T / 2}^{T / 2} a^{2}(t) d t
$$

\subsubsection{Restriction on the PAPR of signal}

The signal $a(t)$ is an even function and the maximum value of signal $a(t)$ is set to be $a(0)$, which is the value of $a(t)$ at time $t=0$. The PAPR of the signal $a(t)$ is considered as a parameter, which has significantly influence on spectral characteristics of the signal $a(t)$; it is defined as:

$$
K=\frac{a_{\max }}{\sqrt{\frac{1}{T} \int_{-T / 2}^{T / 2} a^{2}(t) d t}}=\frac{a(0)}{\sqrt{\frac{1}{T} \int_{-T / 2}^{T / 2} a^{2}(t) d t}}=\frac{a(0)}{\sqrt{\frac{E}{T}}}
$$

Thus, the criterion for minimum out-of-band radiation can be transformed into the problem of unraveling symbol function $a(t)$, when minimizing the Pan-function with the constrains of single-symbol energy, symbol boundary condition, and PAPR of the signal, which can be expressed as follows:

$$
H=J+\lambda\left(\int_{-T / 2}^{T / 2} a^{2}(t) d t-E\right)+\mu\left(a(0)-K \sqrt{\frac{E}{T}}\right)
$$

where $\lambda$ and $\mu$ are the Lagrange constants.

\subsection{Optimized frequency pulse solving process using Fourier series}

Any function in interval $[-T / 2, T / 2]$ can be infinitely approximated by Fourier series $[9,10]$. The efficient 
spectrum signal $a(t)$, with a code width of $T$, can be expressed in the following Fourier series form:

$$
a(t)=\frac{a_{0}}{2}+\sum_{k=1}^{m}\left(a_{k} \cos \left(\frac{2 \pi}{T} k t\right)+b_{k} \sin \left(\frac{2 \pi}{T} k t\right)\right)
$$

where

$$
\begin{aligned}
& a_{0}=\frac{2}{T} \int_{-T / 2}^{T / 2} a(t) d t \\
& a_{k}=\frac{2}{T} \int_{-T / 2}^{T / 2} a(t) \cos \left(\frac{2 \pi}{T} k t\right) d t \\
& b_{k}=\frac{a_{0}}{2}+\sum_{k=1}^{m} a_{k} \cos \left(\frac{2 \pi}{T} k t\right)
\end{aligned}
$$

$a(t)$ is set as an even function; therefore, $b_{k}=0$, and $a(t)$ can be expressed as follows:

$$
a(t)=\frac{a_{0}}{2}+\sum_{k=1}^{m} a_{k} \cos \left(\frac{2 \pi}{T} k t\right)
$$

As $\cos ^{(k)}(t)=\cos \left(t+k \frac{\pi}{2}\right)$, the following equation can be obtained:

$$
\begin{aligned}
a^{(2 n)}(t) & =\sum_{k=1}^{m} a_{k} \cdot\left(\frac{2 \pi}{T} k\right)^{2 n} \cdot \cos \left(\frac{2 \pi}{T} k t+n \pi\right) \\
& =(-1)^{n} \sum_{k=1}^{m} a_{k} \cdot\left(\frac{2 \pi}{T} k\right)^{2 n} \cdot \cos \left(\frac{2 \pi}{T} k t\right)
\end{aligned}
$$

Substituting Eq. (22) into Eq. (15), $J$ can be expressed as follows:

$$
\begin{aligned}
J= & (-1)^{n} \int_{-T / 2}^{T / 2} a(t) a^{(2 n)}(t) d t \\
= & \int_{-T / 2}^{T / 2}\left[\frac{a_{0}}{2}+\sum_{k=1}^{m} a_{k} \cos \left(\frac{2 \pi}{T} k t\right)\right] \\
& \left.\cdot\left[\sum_{k=1}^{m} a_{k} \cdot\left(\frac{2 \pi}{T} k\right)^{2 n} \cdot \cos \left(\frac{2 \pi}{T} k t\right)\right]\right] d t \\
= & \int_{-T / 2}^{T / 2} \frac{a_{0}}{2}\left[\sum_{k=1}^{m} a_{k}\left(\frac{2 \pi}{T} k\right)^{2 n} \cos \left(\frac{2 \pi}{T} k t\right)\right] d t \\
& +\int_{-T / 2}^{T / 2} \sum_{k=1}^{m} a_{k} \cos \left(\frac{2 \pi}{T} k t\right) \cdot\left[\sum_{k=1}^{m} a_{k}\left(\frac{2 \pi}{T} k\right)^{2 n} \cos \left(\frac{2 \pi}{T} k t\right)\right] d t \\
= & \frac{T}{2} \sum_{k=1}^{m} a_{k}^{2}\left(\frac{2 \pi}{T} k\right)^{2 n}
\end{aligned}
$$

where the energy in the signal $a(t)$ is defined as

$$
\begin{aligned}
E & =\int_{-T / 2}^{T / 2} a^{2}(t) d t=\int_{-T / 2}^{T / 2}\left[\frac{a_{0}}{2}+\sum_{k=1}^{m} a_{k} \cos \left(\frac{2 \pi}{T} k t\right)\right]^{2} d t \\
& =\frac{T}{2}\left(\frac{a_{0}^{2}}{2}+\sum_{k=1}^{m} a_{k}^{2}\right)
\end{aligned}
$$

which can be further simplified to obtain:

$$
\frac{2 E}{T}-\left(\frac{a_{0}^{2}}{2}+\sum_{k=1}^{m} a_{k}^{2}\right)=0
$$

Using the Eqs. (23) and (25), (19) can be written as follows:

$$
\begin{aligned}
H= & \frac{T}{2} \sum_{k=1}^{m} a_{k}^{2}\left(\frac{2 \pi}{T} k\right)^{2 n}+\lambda\left(\frac{2 E}{T}-\frac{a_{0}^{2}}{2}-\sum_{k=1}^{m} a_{k}^{2}\right) \\
& +\mu\left(a(0)-K \sqrt{\frac{E}{T}}\right)
\end{aligned}
$$

The normalizations are assumed as $E=1$ and $T=1$; therefore, the coefficients, $a_{0}, a_{1}, \ldots, a_{m}$ of the Fourier series, and the Lagrange constants, $\lambda$ and $\mu$, are the only unknown parameters. The goal is to determine the coefficients, $a_{0}, a_{1}, \ldots, a_{m}$ of the Fourier series. Based on the extreme value theory $[11,12]$ of variational calculus, $a_{0}, a_{1}, \ldots, a_{m}$ should satisfy the following partial differential equations:

$$
\frac{\partial H}{\partial a_{k}}=0 \quad k=1,2, \ldots, m ; \quad \frac{\partial H}{\partial \lambda}=0 ; \quad \frac{\partial H}{\partial \mu}=0 .
$$

The solution of simultaneous equations is used to determine the unknown coefficients.

The Pan-function is solved by using Fourier series, and the solution can be very close to real value when the number of components in Fourier series is infinite. The results computed using MATLAB show that a proper selection of number of components in the Fourier series can guarantee the accuracy of the Panfunction solution. The mean square error $\varepsilon(m)$ is defined as

$$
\varepsilon(m)=\left|a_{m}(t)-a_{m-1}(t)\right|^{2}
$$

where $a_{m}(t)$ is the solution of the Pan-function when the number of components in Fourier series is $m$. Similarly, $a_{m-1}(t)$ is the solution with $m-1$ number of components in the Fourier series. $\varepsilon(m)$ shows the proximity of $a_{m}(t)$ and $a_{m-1}(t)$. When the value of $m$ is equal to 4 , the calculated results indicate an error $\varepsilon(m)$ less than $10^{-5}$. Table 1 
Table 1 Coefficient of Fourier series and signal PAPR

\begin{tabular}{clllllll}
\hline$m$ & $n$ & $a_{0}$ & $a_{1}$ & $a_{2}$ & $a_{3}$ & $a_{4}$ & -0.0306 \\
\hline & 1 & 1.7550 & 0.6627 & -0.1291 & 0.0551 & -0.0021 & 1.44 \\
& 2 & 1.6606 & 0.7875 & -0.0341 & 0.0066 & 0.59 \\
4 & 1.5076 & 0.91996 & 0.12822 & -0.0285 & 0.0094 & 1.79 \\
& 3 & 1.4720 & 0.9396 & 0.1830 & -0.0174 & 0.0032 & -0.0088 \\
& 4 & 1.3519 & 0.98337 & 0.3442 & 0.0281 & -0.0060 & 2.02 \\
& 5 & 1.3475 & 0.9897 & 0.3624 & 0.0404 & 2.06 \\
\hline
\end{tabular}

shows the obtained coefficient of Fourier series and the PAPR under different values of $n$, and $m$. Figure 2 shows the waveforms of the optimized signal $a(t)$ corresponding to different values of $n$.

The optimized signals $a(t)$ have excellent spectral characteristics. Therefore, smooth phase trajectories of CPM signal can be achieved using pulse $a(t)$ as frequency pulse. The obtained CPM signal using proposed method has the characteristics of a higher out-of-band spectral roll-off rate and spectral efficiency.

Considering a normalized solution, the frequency pulse integrates to $1 / 2$ over a symbol interval. Therefore, a normalization coefficient $K$ is required to satisfy

$$
q(t)= \begin{cases}\int_{0}^{t} K a(\tau) d \tau & 0<t<T \\ 1 / 2 & t \geq T\end{cases}
$$

Figure 3 shows the phase pulse $q(t)$ generated by proposed optimized frequency pulse over a symbol interval. The other commonly used phase pulses generated by rectangular pulse, half-sine pulse, and raised cosine pulse are illustrated to provide a comparison with the proposed pulse. It can be noticed that the phase pulse trajectory of proposed optimized frequency pulse is very smooth.
The power spectra of the CPM signal with different frequency pulses are calculated and the normalized power spectra are shown in Fig. 4. The CPM signals with the proposed frequency pulse and the raised cosine pulse have high bandwidth efficiency when the normalized power spectra decrease to $-60 \mathrm{~dB}$. Furthermore, the normalized power spectra of proposed optimized CPM signal decrease more rapidly compare to the other CPM signals when the normalized power spectra attenuation is below $-60 \mathrm{~dB}$. For example, if the bandwidth is compared when the normalized spectra decrease to $-80 \mathrm{~dB}$, the CPM signals with frequency pulses of half-sine pulse, raised cosine pulse, and optimized pulse show the bandwidths of $7.8 / T, 5.3 / T$, and $4.6 / T$, respectively. While the normalized power spectra decrease to $-90 \mathrm{~dB}$, the CPM signals with frequency pulses of raised cosine pulse and optimized pulse show the bandwidths of $6.8 / T$ and $5 / T$, respectively. Thus, the obtained optimized CPM signal can well inhibit the out-of-band energy radiation of the signal.

A part of the phase tree for obtained CPM signal with optimized frequency pulse is illustrated in Fig. 5. For comparison, the corresponding phase tree for MSK is also shown.

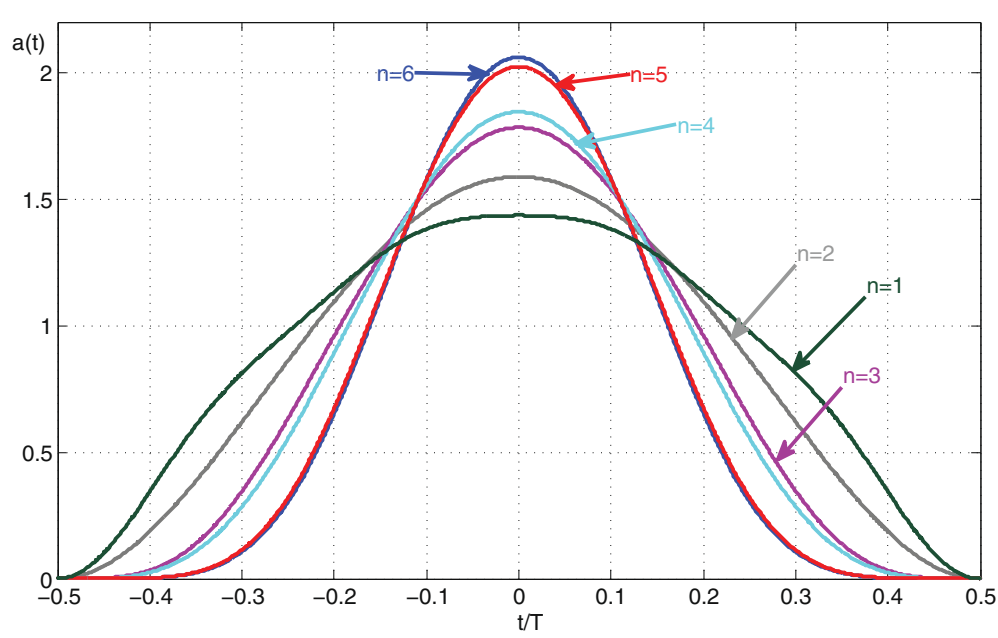

Fig. 2 Waveform curves of the optimized signals 


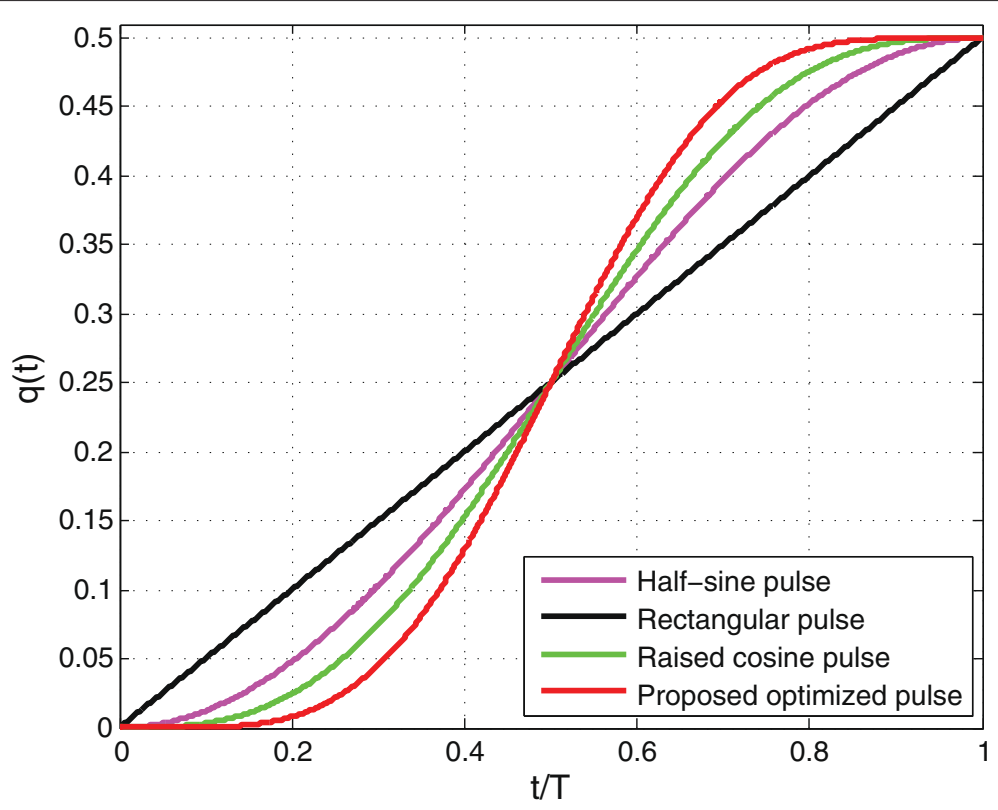

Fig. 3 Phase pulses for full response CPM scheme with different frequency pulses

The power spectra of the CPM signals selecting different frequency pulses under different values of $n$ and PAPR are calculated, according to Table 1.

The normalized power spectra density of the obtained CPM and MSK signals are illustrated in Fig. 6, and it can be observed that the power spectra of obtained optimized signal fall off considerably faster. For example, if the bandwidth is compared when the normalized power spectra decrease to $-60 \mathrm{~dB}$, the optimized CPM signal show a bandwidth of $3.2 / T$ (when $n=3, m=4$ ), while the bandwidth is decreased by 2.43 times compared to the MSK signal, thus well inhibiting the out-of-band energy radiation of signals. Further bandwidth efficiency can be achieved by increasing the value of $n$; therefore, the out-of-band radiation of signals and the interference for adjacent channel will be significantly reduced.

\section{The modulation and demodulation scheme}

\subsection{The modulation scheme of system}

A CPM signal can be expressed by two orthogonal components:

$s_{k}(t)=\sqrt{\frac{2 E_{s}}{T}}\left\{p_{k} C(t) \cos 2 \pi f_{c} t+q_{k} S(t) \sin 2 \pi f_{c} t\right\}$
$(k-1) T<t \leq k T$

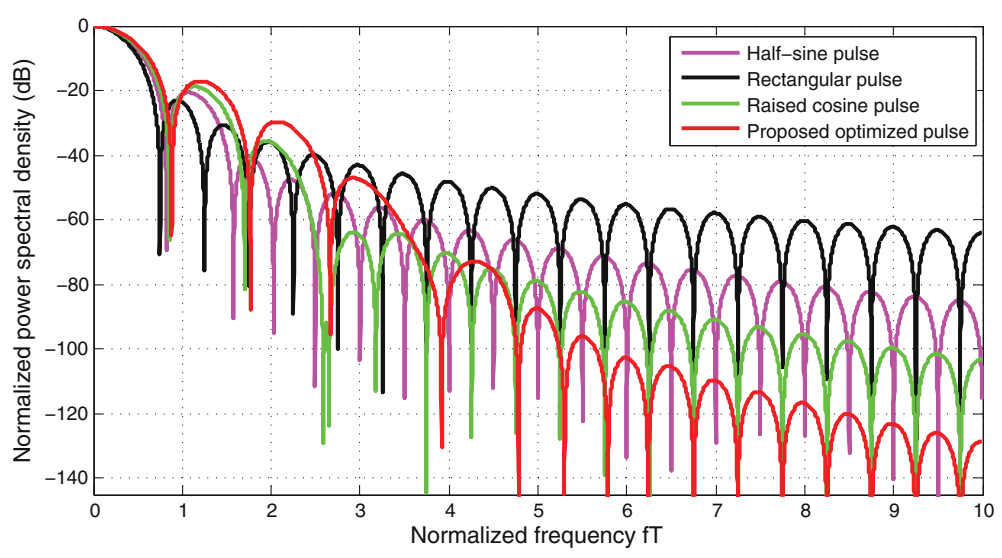

Fig. 4 Power spectra for different frequency pulses 


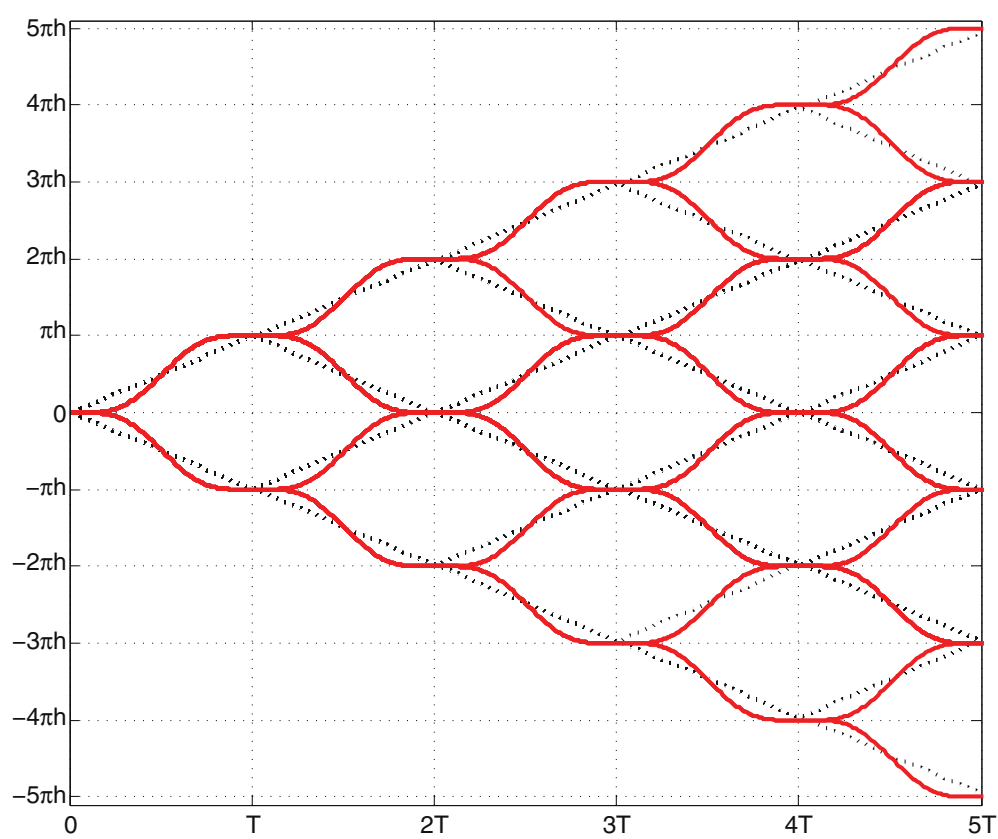

Fig. 5 Phase tree for MSK (dashed) and binary, full response CPM with optimized frequency pulse (solid)

where $C(t)$ and $S(t)$ are defined as:

$$
\begin{aligned}
& C(t)=\cos \left\{2 \pi h \sum_{n=-\infty}^{\infty} q(t-n T)\right\} \\
& S(t)=\sin \left\{2 \pi h \sum_{n=-\infty}^{\infty} q(t-n T)\right\}
\end{aligned}
$$

Block diagram of the quadrature modulation system is shown in Fig. 7. The input data sequence $a_{k}$, is transformed into sequence $b_{k}$ after differential encoding. Afterwards, the sequence $b_{k}$ is converted parallel, and the even data are sent from the upper branch $p_{k}$, while the odd data are sent from the lower branch $q_{k}$. Note that the durations of symbols after serial to parallel converter is $2 T$.

\subsection{The demodulation scheme of system}

The normalized minimum squared Euclidean distance of $h=1 / 2$ binary full response CPM signals with the frequency pulses of proposed optimized frequency, rectangular pulse, half-sine pulse, and raised cosine pulse that are calculated refer to [1]. And all of them have

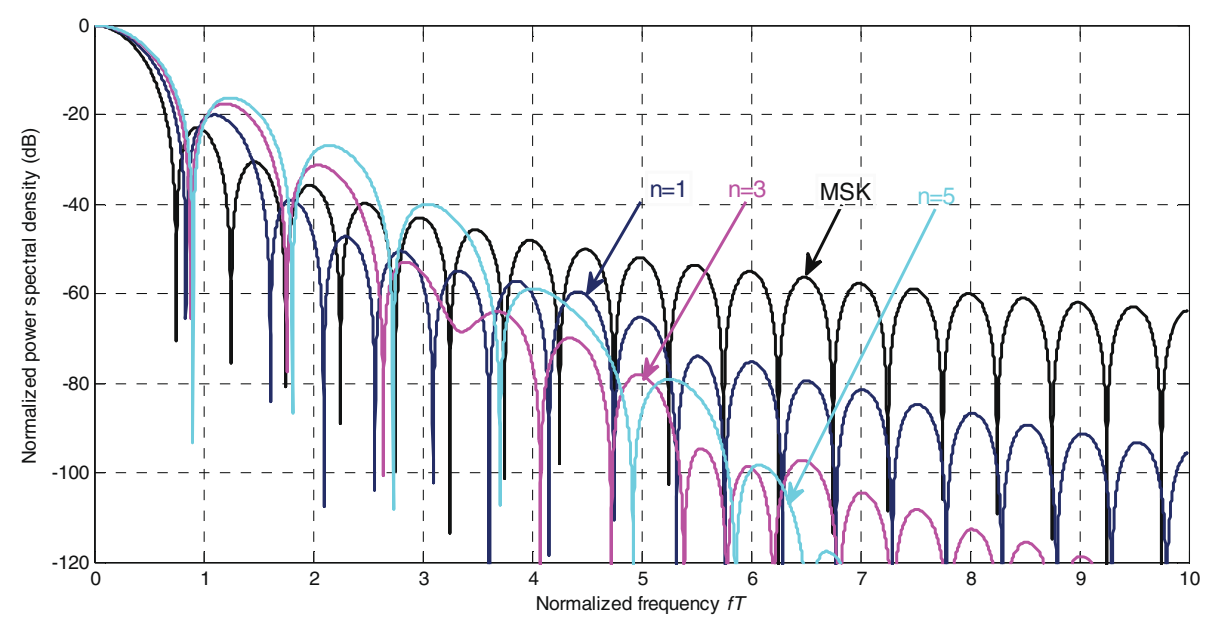

Fig. 6 Power spectra of obtained CPM signal with optimized frequency pulse and MSK 


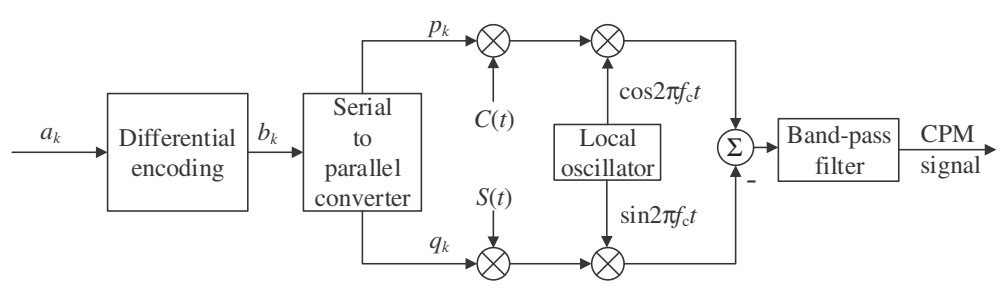

Fig. 7 Block diagram of the quadrature modulation system

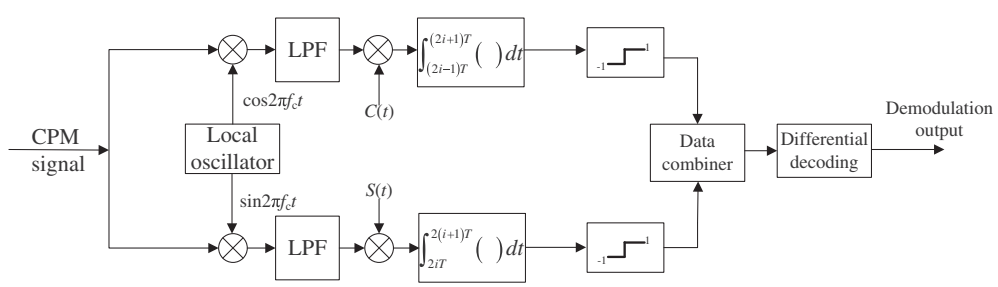

Fig. 8 Block diagram of the optimum receiver

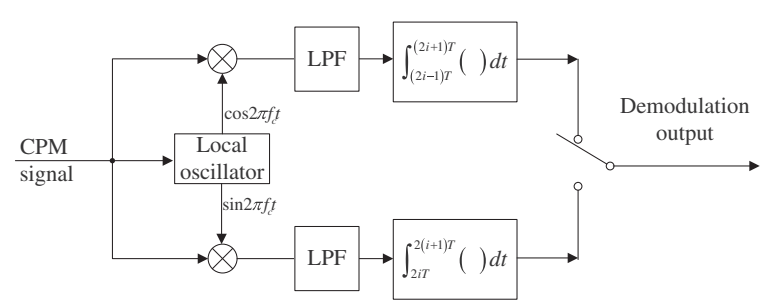

Fig. 9 Block diagram of the reduced-complexity receiver
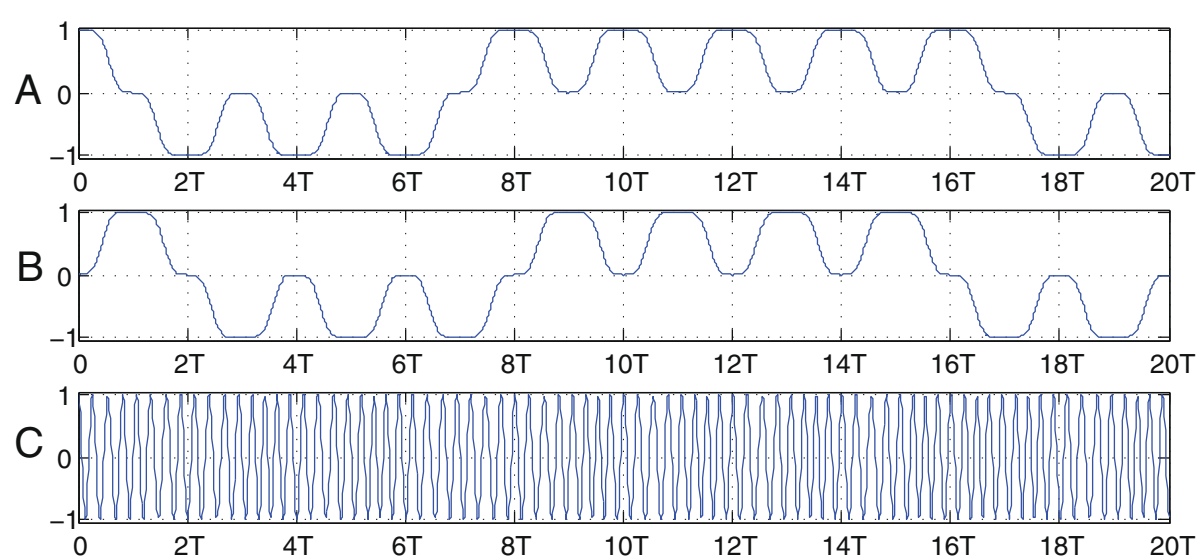

Fig. 10 Simulation results of the quadrature modulation 

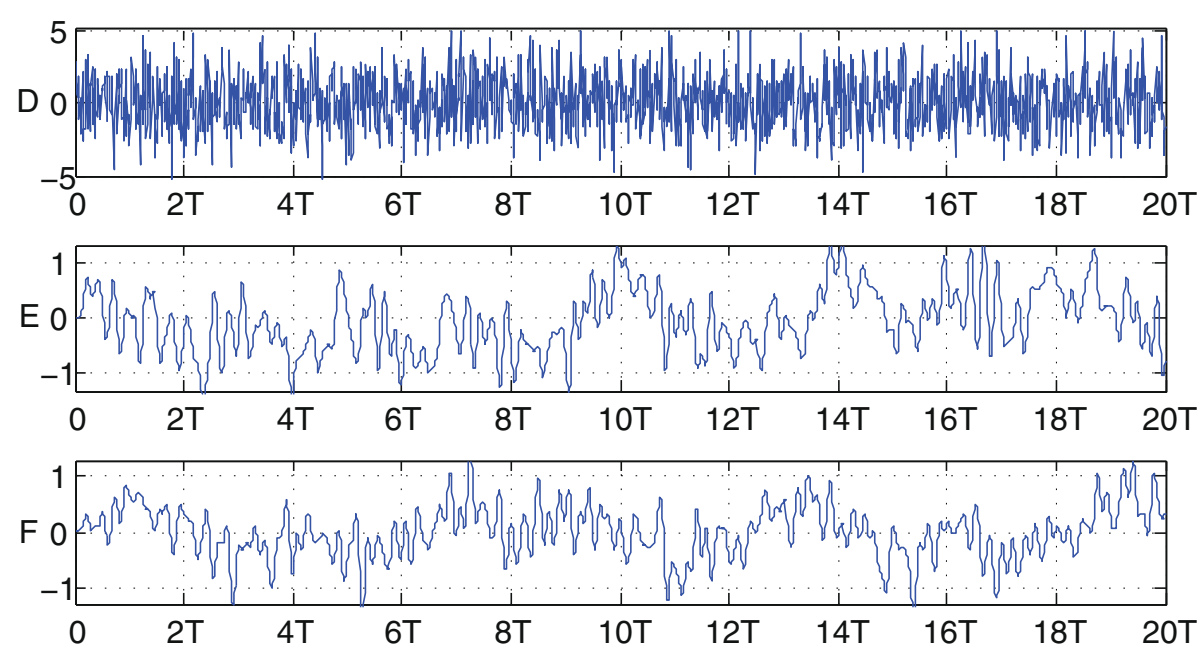

Fig. 11 Simulation results of the coherent demodulation $\left(E_{b} / N_{0}=10\right)$

the minimum squared distance $d_{\mathrm{min}}^{2}=2$. Therefore, the bit error rate (BER) performance of CPM with frequency pulse of optimized pulse is comparable to that of raised cosine pulse and rectangular pulse. However, the optimized CPM signal has a higher spectral roll-off rate and less out-of-band energy radiation well inhibiting the adjacent channel interference. Block diagram of the optimum receiver is shown in Fig. 8. The CPM signals with frequency pulses discussed above can have almost the same BER performance when the optimum receiver is adopted. In order to reduce receiver complexity, a reduced-complexity receiver is used to complete the demodulation process shown in Fig. 9. The received modulated signal is coherently demodulated by the quadrature carriers and low-pass filters to remove the high-frequency components. The signal on each channel is integrated, and the baseband signal is acquired by sampling.

\subsection{Simulation of modulation and demodulation}

The signal obtained with $n=3, m=4$, was used as the baseband symbol, and the simulations of quadrature modulation and coherent demodulation were completed using the MATLAB software. The simulation results are shown in Figs. 10 and 11.

In Fig. 10, A is the baseband symbol signal transmitted in the in-phase channel, B is the baseband symbol signal transmitted in the orthogonal channel, and $\mathrm{C}$ is the quadrature-modulated output signal.

Figure 11 shows the simulation results of coherent demodulation when the $E_{b} / N_{0}$ is equal to $10 \mathrm{~dB}$. In Fig. 11, D is the transmitted signal corrupted with additive white Gaussian noise (AWGN), which is the input to the receiver, $\mathrm{E}$ is the baseband signal demodulated in the in-phase channel, and F is the baseband signal demodulated in the orthogonal channel. The envelope of the output signal after the modulation is found to be constant and consistent with the expected results.

In Fig. 12, the BER performance of the reducedcomplexity scheme with AWGN is presented. The performance of CPM signal with frequency pulses of rectangular pulse (MSK) and raised cosine pulse are also shown for comparison; these are strong competitors of the proposed scheme. It can be noticed from Fig. 12 that the required $E_{b} / N_{0} \mathrm{~s}$ are $7.8,8.3$, and 8.6, to achieve a $\mathrm{BER}=10^{-3}$ for rectangular pulse, raised cosine, and proposed pulse, respectively. And we can observe that the optimized CPM signal is only about $0.7 \mathrm{~dB}$ inferior to CPM signals with frequency pulses of rectangular pulse (MSK) at a BER of $10^{-4}$. Although the BER

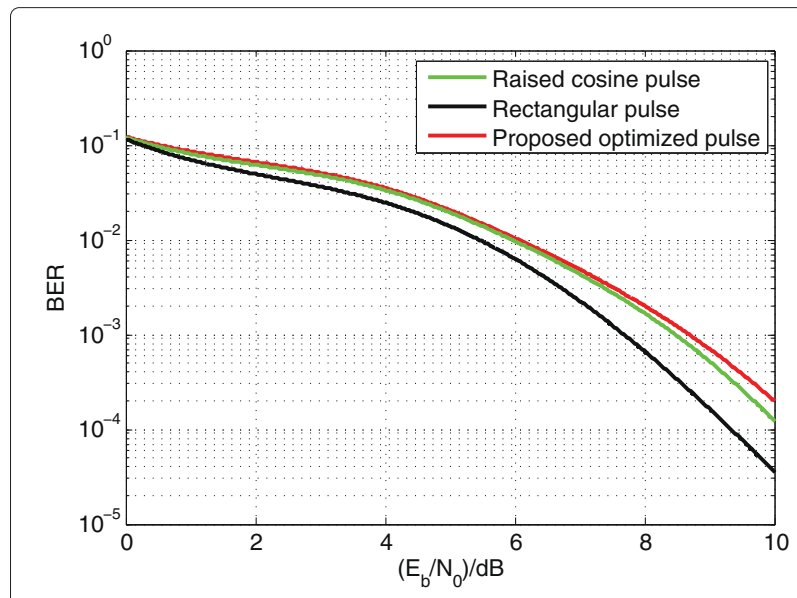

Fig. 12 Bit error rate curves of simulation system 
performance suffers a small $E_{b} / N_{0}$ penalty which is a small price to pay for the large improvement in bandwidth efficiency and reduction in implementation complexity. Additional gains in performance can be achieved by introducing additional redundancy through coding. In particular, trellis-coded CPM using relatively simple convolution codes has been thoroughly investigated and many results are available in the technical literature. The Viterbi decoder for the convolutionally encoded CPM signal now exploits the memory inherent in the code and in the CPM signal. Performance gains of the order of 4$6 \mathrm{~dB}$, relative to uncoded MSK with the same bandwidth, have been demonstrated by combining convolutional coding with CPM. Also, the detector is purely an integrateand-dump (I\&D) (i.e., matched to a rectangular pulse); the received signal plus noise is respectively followed by $I \& D$ circuits of duration $2 T$. The multiplier-integrator combination constitutes a matched filter that, in the case of AWGN and no intersymbol interference (ISI), results in optimum detection. When optimum detection is adopted, the system could also obtain performance gains. The complexity of demodulation scheme adopted in this paper is very small and equivalent to normal QPSK or OQPSK demodulation scheme with a low cost in hardware implemention. So the proposed optimized pulse is the most suitable method due to its superior spectral efficiency and lower complexity. As discussed above, the method proposed in this paper can be employed in bandlimited channel with high requirement for adjacent channel out-of-band energy radiation and completed in low complexity.

\subsection{Hardware implementation}

The modulation and demodulation of the CPM system is ultimately implemented on the field programmable gate array (FPGA) devices. FPGA have features of high integration, flexible programming, more pins, low power, and fast design speed. The hardware architectures have been implemented in Quartus II software which provides several tools for synthesizing the design, configuration techiques, performance analyses, including resource, speed, and power consumption. The whole system is divided into several small modules based on top-down design method and using verilog HDL hardware description language to design each module. The whole system also has been simulated in Modelsim SE10.0 simulation environment and successfully downloaded to the chip of the Altera Cyclone III EP3C55U484C6N.

According to the block diagram of the quadrature modulation system shown in Fig. 7, the architecture has been built with some basic modules in Quartus II. It has realized differential coding, serial-to-parallel converter, and modulation. Figure 13 shows the simulation waveforms of CPM modulation signal under Modelsim SE10.0. "Baseband_I" is the baseband symbol signal transmitted in the in-phase channel, "Baseband_Q" is the baseband symbol signal transmitted in the orthogonal channel, and "Modulated signal" is the modulated CPM signal; we can observe that the envelope of the modulated signal is found to be constant.

According to the block diagram of the reducedcomplexity receiver in Fig. 9, the I\&D circuits are timed to match the zero crossings of the I and Q symbol waveforms and easy to complete. However, carrier synchronization is very important and difficult in this scheme. In carrier synchronization module, the received signal multiplies the quadrature carriers generated from local oscillator with the same frequency and phase as the carrier of transmitter. The carrier is generated by the module of NCO. Figure 14 shows the simulation waveforms of baseband signal output after carrier synchronization. "Demodulated_I" is the baseband symbol signal demodulated in the in-phase channel, "Demodulated_Q" is the baseband symbol signal demodulated in the orthogonal channel, and "Phase discriminator" is the phase discriminator output; we can observe that the carrier synchronization can be fulfilled, and the phase locked is accomplished.

For high carrier frequencies, direct synthesis of the CPM signal by computing phase, using a digital approach is impractical since maintaining an adequate sampling rate requires an extremely high operating frequency. Instead, one can resort to a quadrature implementation, where baseband I and Q signals containing the phase

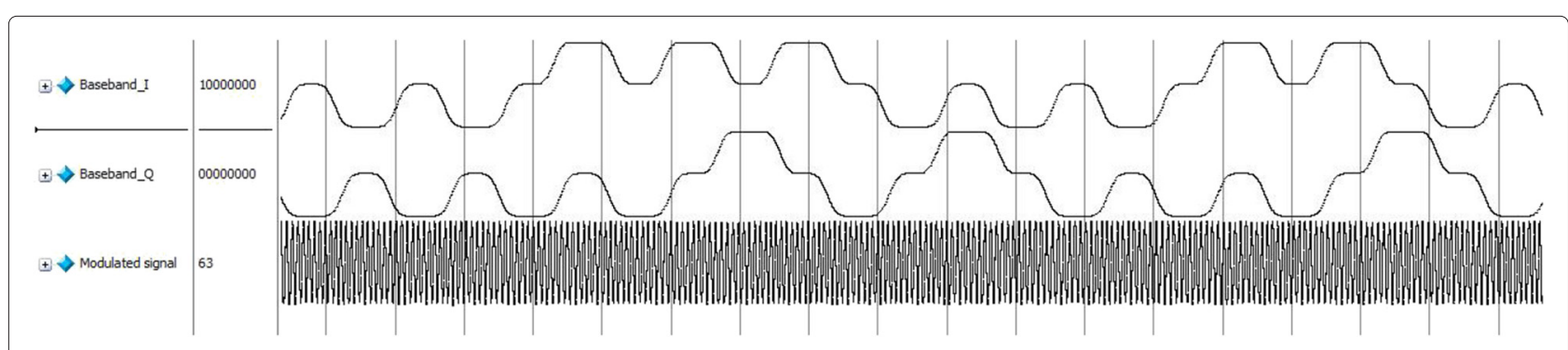

Fig. 13 Waveforms of CPM modulation signal 


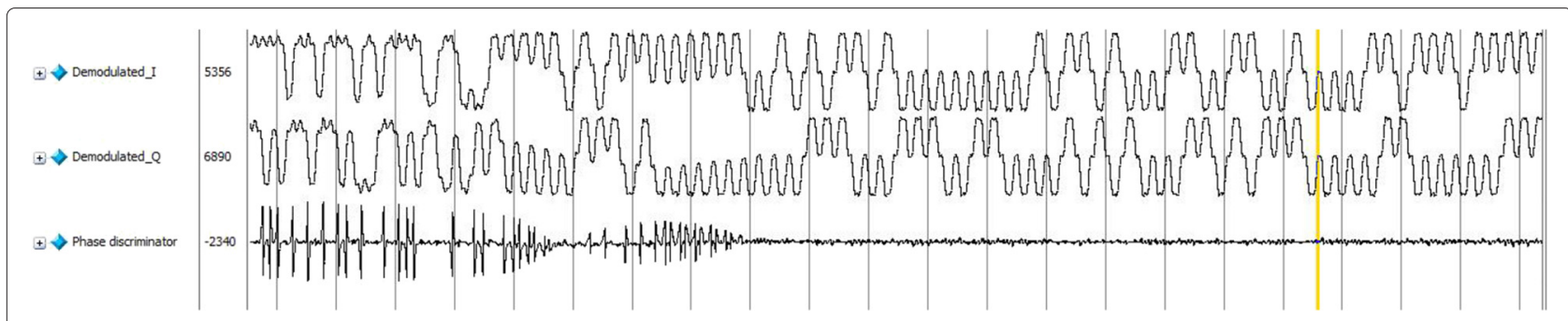

Fig. 14 Waveforms of baseband signal output after carrier synchronization

information are generated that vary much slower than the phase of the modulated carrier, thus making it feasible to implement them digitally. An efficient I-Q implementation of a CPM modulator skips some steps and instead generates the I and $\mathrm{Q}$ baseband signals directly from the binary data, thereby eliminating errors in filtering and phase and sine/cosine computation inherent in the conventional architecture.

In band-limited communication system, the modulated signal always run through filter to limit the bandwidth of the signal; however, the hardware resource consumption of filter includes the multipliers, adders, and registers. In this paper, the binary data output is passed through optimized waveform read-only memories (ROMs) whose outputs are applied to I and Q carriers. Also, the modulated signal passing through filter could introduce ISI and increase the complexity of the channel equalization.
When optimized symbol waveform is employed, the modulation signal has a more compact power spectrum; therefore, a signal does not need to be filtered to reduce the out-of-band power radiation which can reduce system complexity and hardware sources. The scheme applied in this paper could obtain significant hardware reduction. Meanwhile, we can collocate hardware resources reasonably, for example, we can select the number of sampling points within a symbol period according to the hardware sources. The higher the number of sampling points is, the higher accuracy of the output waveform would be. For example, we can sample 64 points within a symbol period or more points to store in ROMs. The method of ROM table lookup can cause $\mathrm{T}$ time delay; however, it has little influence for high-speed information transfer communication system. The experimental setup of the CPM system is shown in Fig. 15.

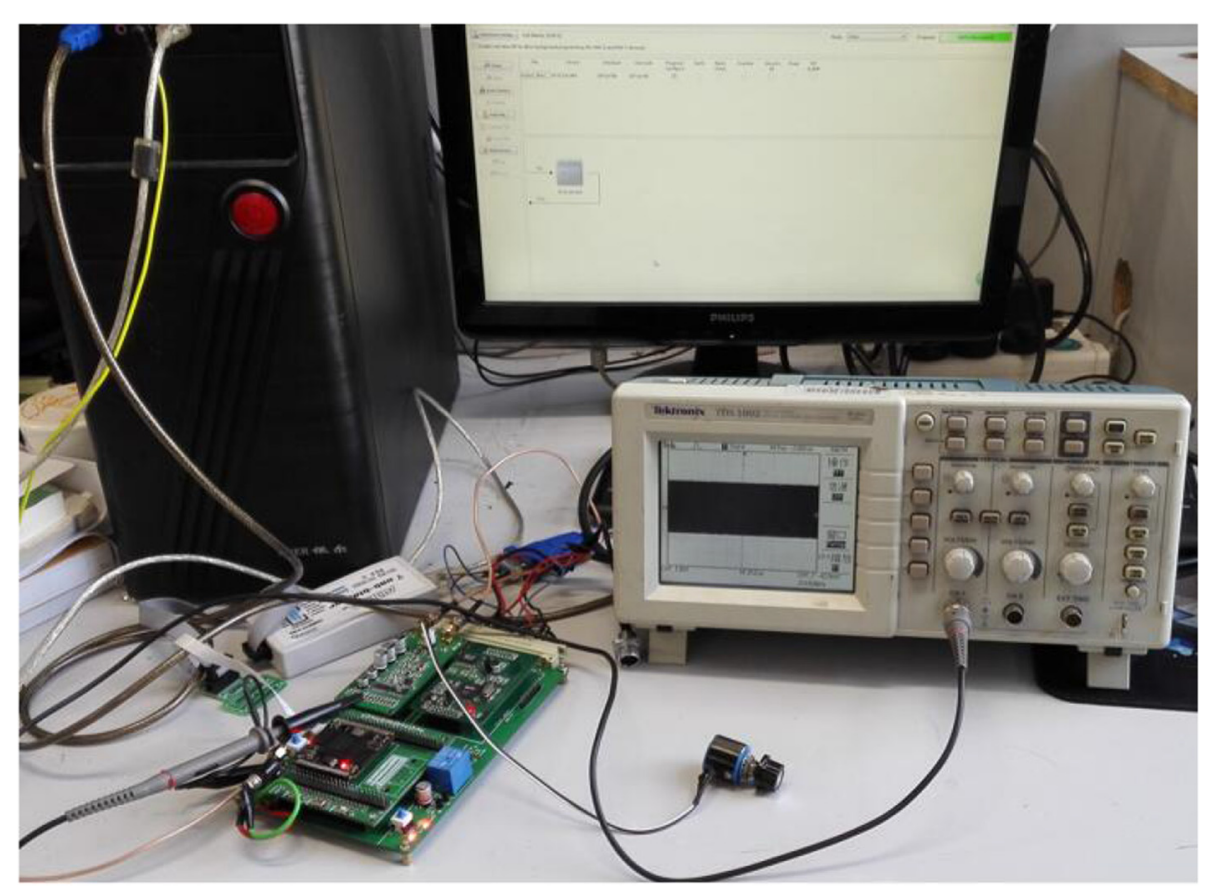

Fig. 15 Experimental setup of the CPM system 


\section{Conclusions}

In general, the constant continuous phase modulation systems use the pre-existing waveforms as frequency pulse, such as rectangular, cosine, raised cosine pulse, and other forms or through a filter (GMSK signal). There are few uses of the method of establishing a mathematical model base on some standards to obtain the optimized frequency pulse. In this paper, a Pan-function model is established to obtain optimized frequency pulse in accordance with the minimum out-of-band energy radiation criterion. The mathematical derivation is performed using Fourier series. It is shown that the phase trajectories of the CPM signal are smoothed using the proposed frequency pulse. The simulation of the signal modulation and demodulation scheme was performed using the MATLAB software. It is demonstrated that the CPM signal with proposed optimized frequency pulse has a higher spectral roll-off rate, less out-of-band radiation, and a constant envelope. The whole system has been successfully downloaded to FPGA devices. The operating results are consistent with expected results and it is verified the correctness of this method.

\section{Competing interests}

The authors declare that they have no competing interests.

\section{Acknowledgements}

This research has been supported by International Science and Technology Cooperation Program of China (2014 DFR10240), Harbin Science and Technology research projects (2013AE1BE003), Hei Long Jiang Postdoctoral Foundation (LBH-Z14066), and the Fundamental Research Funds for the Central Universities (China) [HEUCF1508].

\section{Author details}

${ }^{1}$ College of Information and Communication Engineering, Harbin Engineering University, 150001, Harbin, China. ${ }^{2}$ Institute of Physics, Nanotechnology and Telecommunications, St. Petersburg State Polytechnical University, 195251 St. Petersburg, Russia.

Received: 20 July 2015 Accepted: 18 April 2016

Published online: 05 May 2016

\section{References}

1. JG Proakis, DG Manolakis, Digital Signal Processing: Principles Algorithms and Applications. (Prentice Hall, New Jersey, 1996)

2. A Barbieri, D Fertonani, G Colavolpe, Spectrally-efficient continuous phase modulations. IEEE Trans. Wirel. Commun. 8(3), 1564-1572 (2009)

3. E Doyuran, Y Tanik, in Vehicular Technology Conference, 2006. VTC 2006-Spring. IEEE 63rd. New pulse shapes for CPM signals, vol. 1 (IEEE, Melbourne, Australia, 2006), pp. 236-240

4. R-H Yang, M-T Lee, C-K Lee, S-J Chern, in Intelligent Signal Processing and Communications Systems (ISPACS), 2011 International Symposium On. A novel class of continuous-phase modulation (CPM) with separable phase property (IEEE, Chiang Mai, Thailand, 2011), pp. 1-6. http://dx.doi.org/10. 1109/ISPACS.2011.6146148

5. LA SHKOL'NYY, The minimum out-of-band radiation optimization of radio pulse shape. Radio Technol. 30(06), 12-15 (1975)

6. W Xue, W Ma, B Chen, in 6th International Conference on Wireless Communications, Networking and Mobile Computing, WiCOM. A realization method of the optimized efficient spectrum signals using Fourier series, IEEE Antennas and Propagation Society; IEEE Communications Society; Southwest Jiaotong University; University of Electronic Science and Technology of China; Wuhan University, Chengdu, China, (2010). http:// dx.doi.org/10.1109/WICOM.2010.5600972
7. Z Dong, Proficient in Programming and Database Applications of MATLAB7. (Publishing House of Electronics Industry, Beijing, 2007)

8. MK Simon, Bandwidth-efficient Digital Modulation with Application to Deep-space Communications, vol. 2. (Wiley, Hoboken, 2005)

9. J Zheng, Q Ying, W Yang, Signals And Systems. (Higer Education Press, Beijing, 2000)

10. SB Makarov, W Xue, Synthesis of spectrally-efficient signals for secure wireless telecommunication systems with limited energy resource. Inf. Secur. Technol. Comput. Syst. 2, 83-94 (2006)

11. LD Elsgolc, Calculus of Variations. (Courier Dover Publications, Mineola, 2012)

12. JL Synge, The Hypercircle in Mathematical Physics: a Method for the Approximate Solution of Boundary Value Problems. (Cambridge University Press, New York, 2012)

\section{Submit your manuscript to a SpringerOpen ${ }^{\circ}$ journal and benefit from:}

- Convenient online submission

- Rigorous peer review

- Immediate publication on acceptance

- Open access: articles freely available online

- High visibility within the field

- Retaining the copyright to your article

Submit your next manuscript at $\mathbf{s p r i n g e r o p e n . c o m ~}$ 